\title{
Effects of obstetric factors and storage temperatures on the yield of endothelial colony forming cells from umbilical cord blood
}

\author{
Kate E. Coldwell $\cdot$ Stephanie J. Lee $\cdot$ \\ Jennifer Kean • Cheen P. Khoo • Grigorios Tsaknakis • \\ Jon Smythe · Suzanne M. Watt
}

Received: 3 May 2011/Accepted: 16 June 2011/Published online: 1 July 2011

(C) The Author(s) 2011. This article is published with open access at Springerlink.com

\begin{abstract}
As umbilical cord blood (UCB) is a rich source of endothelial colony-forming cells (ECFC), our aim was twofold: (1) to examine potential obstetric selection criteria for achieving the highest ECFC yields from UCB units, and (2) to determine whether transient storage temperatures of fresh UCB and cryopreservation of UCB units affected ECFC yield and function. ECFC quality was assessed before and after cryopreservation by their clonogenic proliferative potential. Of the 20 factors examined, placental weight was the only statistically significant obstetric factor that predicted ECFC frequency in UCB. Studies on the effects of storage revealed that transient storage of fresh UCB at $4^{\circ} \mathrm{C}$ reduced ECFC yield compared with storage at $22^{\circ} \mathrm{C}$, while cryopreservation of UCB MNCs significantly reduced ECFC recoveries. To our knowledge, this is the first demonstration that placental weight and temperature of storage prior to processing or culture have significant effects on ECFC frequency in UCB. Our studies further support the evidence that cryopreservation of UCB MNCs compromises ECFC recovery.
\end{abstract}

\footnotetext{
K. E. Coldwell $(\bowtie) \cdot$ S. J. Lee $\cdot$ J. Kean .

C. P. Khoo - G. Tsaknakis - J. Smythe - S. M. Watt

Stem Cell Research Laboratory, NHS Blood and Transplant, Oxford OX3 9BQ, UK

e-mail: kate.e.coldwell@gmail.com

\section{S. J. Lee}

e-mail: stephlee11@gmail.com

J. Kean

e-mail: jenniferkean@nhs.net

C. P. Khoo

e-mail: Cheen.Khoo@nhsbt.nhs.uk

G. Tsaknakis

e-mail: grigorios.tsaknakis@gmail.com

J. Smythe

e-mail: Jon.Smythe@nhsbt.nhs.uk
}

Keywords Umbilical cord blood - Endothelial progenitor cells · Cryopreservation · Endothelial colony-forming cells $\cdot$ Transient storage $\cdot$ Cord blood banking

$\begin{array}{ll}\text { Abbreviations } \\ \text { ECFC } & \text { Endothelial colony forming cells } \\ \text { UCB } & \text { Umbilical cord blood } \\ \text { MNC } & \text { Mononuclear cell } \\ \text { CBB } & \text { Cord blood bank } \\ \text { TNC } & \text { Total nucleated cell content } \\ \text { HSC } & \text { Hematopoietic stem cells } \\ \text { HPC } & \text { Hematopoietic progenitor cells } \\ \text { DNase } & \text { Deoxyribonuclease }\end{array}$

\section{Introduction}

Umbilical cord blood (UCB) is increasingly used as an alternative source of hematopoietic stem and progenitor

\author{
S. M. Watt \\ e-mail: Suzanne.Watt@nhsbt.nhs.uk \\ S. J. Lee · J. Kean · S. M. Watt \\ Nuffield Department of Clinical Laboratory Sciences, University \\ of Oxford, Oxford OX3 9DU, UK \\ S. J. Lee \\ Medical Sciences Division, University of Melbourne, Melbourne \\ 3050, Australia \\ J. Kean \\ Department of Plastic and Reconstructive Surgery, Stoke \\ Mandeville Hospital, Aylesbury HP21 8AL, UK
}


cells (HSC/HPC) to bone marrow and mobilized peripheral blood for HSC transplantation in both children and adults $[1,2]$. It has the advantage that it can be tissue typed, screened for viral biomarkers, processed and banked, especially ahead of urgent or directed transplants, without the attrition rate that occurs with bone marrow donors [1-4]. Of note, a recent report states that strategic objectives for the UK NHSBT Cord Blood Bank (CBB) are maximized clinical benefits and inventory utilization where a feasible target is $1 \%$ annual utilization of banked UCB units [5].

UCB and the umbilical cord itself have been reported to contain other stem/progenitor cell types, including mesenchymal stem/stromal cells and endothelial progenitor cells [late endothelial outgrowth cells or endothelial colony forming cells (ECFC)]. These overall types may have the potential to improve transplant outcomes when used in conjunction with HSC $[6,7]$. Although ECFC are found in umbilical cord tissue (HUVEC) and UCB at higher levels and with higher proliferative potential than in adult peripheral blood [8-10], factors affecting their yield and recovery from neonatal sources are still not understood. In contrast, since the first UCB HSC transplant in 1988 [11], a great deal of knowledge has been acquired on the optimal selection of UCB units for HSC transplantation [12, 13]. This is based on obstetric factors (Table 1), total nucleated cell content (TNC), $\mathrm{CD} 4^{+}$cell count, HLA and blood group matching of the recipient and donor plus processing, storage and testing procedures. Other quality measurements such as maternal HLA typing to assess the donor's non-inherited maternal HLA-antigens may also influence transplant success [1, 2, 12]. Typically, UCB is collected from a wide range of donors, held for a period of time (not exceeding 24-48 h) at $22 \pm 2{ }^{\circ} \mathrm{C}$ prior to processing, and then cryopreserved under controlled rate freezing conditions and stored long-term below $-150^{\circ} \mathrm{C}$ (as recommended by international accreditation agencies such as FACT-Netcord) $[1,2,8]$. Units are also analyzed before or after processing for $\mathrm{TNC}, \mathrm{CD}_{3}{ }^{+}$cell numbers and hematopoietic colony forming units [1, 8, 12-14]. Obstetric factors that have previously been reported to affect TNC and/or HSC/HPC $\left(\mathrm{CD} 34^{+}\right.$cells and total CFU) content include birth and placental weight, infant gender, parity of the mother, nucleated red cell content, low venous $\mathrm{pH}$, prolonged first stage of labor and Apgar scores [quantifies the ability of the infant to adapt to the extra uterine environment and hence a measurement of the health of newborn infants at 1,5 and 10 min post birth $\left(\right.$ Apgar $_{1 \mathrm{~min}}$, Apgar $\left._{5 \mathrm{~min}}, \operatorname{Apgar}_{10 \mathrm{~min}}\right)$ with the normal Apgar score varying from 7 to 10].

It remains uncertain how this knowledge of UCB HSC/ HPC quality and storage affects the late endothelial outgrowth cell/ECFC content of UCB. A recent report indicates that pre-term UCB units at 28-35 weeks of gestation contain $\sim$ fourfold more ECFCs than term UCB [15], yet no other obstetric features were examined. Additionally, studies on ECFC recovery after UCB cryopreservation are conflicting, with Lin et al. [16] indicating that the outgrowth endothelial cell content is not statistically different between cryopreserved and fresh UCB mononuclear cells although on average fewer were recovered after cryopreservation, while Vanneaux et al. [17] recovered significantly fewer ECFC from cryopreserved than from fresh UCB units.

In order to achieve optimal ECFC yields from UCB at term deliveries, we set out to determine (1) how to best select UCB donors based on obstetric factors, (2) whether storage temperatures of UCB units using current standards for UCB HSC/HPC of $22 \pm 2{ }^{\circ} \mathrm{C}$ prior to processing was superior to storage at $4^{\circ} \mathrm{C}$, and (3) whether ECFC content was compromised following cryopreservation. As with HSC/HPC transplants, an understanding of optimal donor selection, short-term storage conditions of whole UCB before processing and long-term storage conditions of cryopreserved UCB may in the future enhance the efficacy of UCB ECFC for therapeutic use.

\section{Materials and methods}

\section{Materials}

Wash buffer A consisted of Hank's buffered salt solution (HBSS) without calcium and magnesium (Lonza Biologics, Slough, England) supplemented with $3 \mathrm{~g} / \mathrm{l}$ sodium citrate and $0.5 \%(\mathrm{v} / \mathrm{v})$ Hyclone fetal calf serum (FCS; Thermo Scientific, Loughborough, England). Wash buffer B consisted of HBSS without calcium and magnesium (Lonza Biologics). Growth medium ('complete EGM-2') comprised EBM-2 media supplemented with all components of the SingleQuot Bullet Kit [hydrocortisone, human epidermal growth factor, human fibroblast growth factor-basic, insulin-like growth factor-1, vascular endothelial growth factor, ascorbic acid, heparin, gentamicin, 2\% (v/v) FCS] (Lonza Biologics) and then further supplemented with $8 \%$ (v/v) Hyclone FCS and 1\% (v/v) penicillin/streptomycin (PAA Laboratories Ltd., Yeovil, England). Bovine DNase I (Sigma-Aldrich Ltd., Poole, England) was prepared in $50 \%$ (v/v) glycerol, $5 \mathrm{mM} \mathrm{CaCl}_{2}$ in $\mathrm{HBSS}(\mathrm{pH} 7.8)$ and stored at $-20^{\circ} \mathrm{C}$. 'Thaw buffer' consisted of growth media with $10 \mathrm{U} / \mathrm{ml}$ DNase I. Forty eight- and 6-well tissue culture plates pre-coated with type-1 rat tail collagen were purchased from BD Biosciences (Oxford, England). Other cell culture vessels were from Corning (Lowell, MA, USA), $0.5 \%(\mathrm{w} / \mathrm{v})$ trypsin and $0.2 \%(\mathrm{w} / \mathrm{v})$ EDTA (trypsinEDTA) from PAA Laboratories Ltd., $0.4 \%$ (w/v) trypan blue from Invitrogen Ltd. (Paisley, Scotland) and $\mathrm{F}_{\mathrm{C}}$ Receptor Blocking Reagent from Miltenyi-Biotec. (Bergisch-Gladbag, Germany). Unless otherwise specified, 


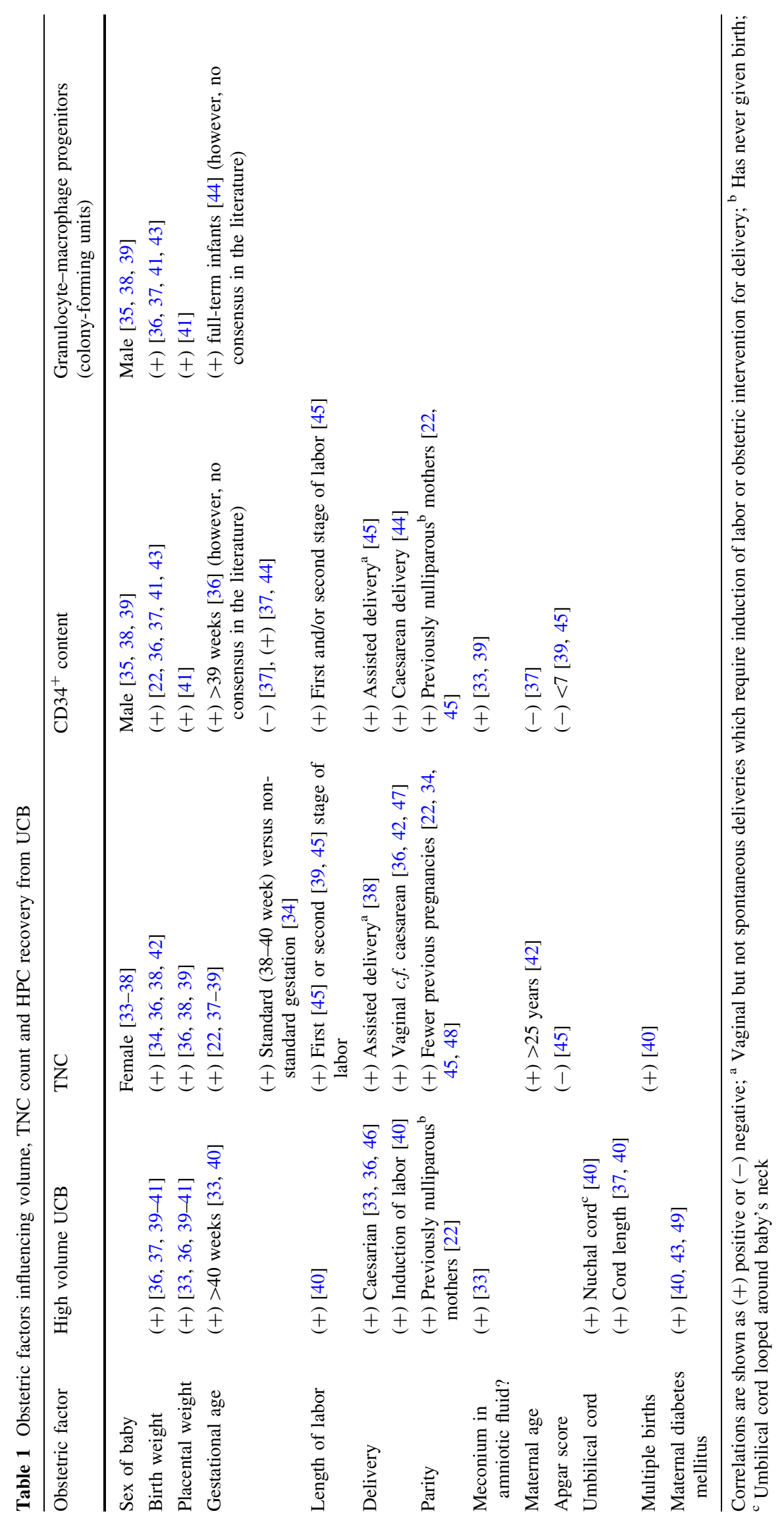


incubations were at $37^{\circ} \mathrm{C}$ in a humidified incubator with $5 \% \mathrm{CO}_{2}$ in air. Accuspin tubes containing Ficoll-Histopaque-1077 (1.077 g/ml), Fungizone and all other materials were obtained from Sigma-Aldrich Ltd.

\section{Umbilical cord blood collection}

Fresh UCB was collected by the NHSBT Cord Blood Resource team (Oxford, England) (termed 'R\&D UCB') with informed written consent from mothers at the John Radcliffe Hospital, Oxford in accordance with Oxfordshire Research Ethics Committee approval. These mothers were over the age of 18 , had uncomplicated births, screened negative at the time of delivery for a range of infections (e.g. HIV, HBV, HCV, syphilis), did not use illegal drugs and were not delivering at prematurity ( $<36$ weeks). Further exclusion criteria were known genetic defects of the baby (e.g. Down's syndrome, Spina Bifida) due to the requirement for diagnostic placental specimens and to reduce maternal distress. Due to other research projects taking place at this hospital, no pre-eclampsic mothers donated to this study. UCB was collected directly from the umbilical cord as soon as possible after delivery of the placenta by needle cannulation into sterile UCB collection bags containing $21 \mathrm{ml}$ citrate phosphate dextrose anticoagulant (MacoPharma Ltd., Twickenham, England). Cell counts from a sample of UCB from the bag were analyzed using a Sysmex total blood analyzer (Sysmex XE-2100, Sysmex UK Ltd., Milton Keynes, England). Samples were anonymised prior to being made available to researchers. Obstetric observations were obtained from archived data by the Oxford-Radcliffe Hospital Information Service by certified staff not involved in the research.

Mononuclear cells (MNC) were isolated from UCB by centrifugation on Ficoll-Histopaque (density $1.077 \mathrm{~g} / \mathrm{ml}$ ) using Accuspin tubes as per the manufacturer's instructions. The resulting buffy coat was resuspended in wash buffer $\mathrm{A}$ and sedimented once at $400 \times g$ to remove Ficoll, once at $200 \times g$ to reduce platelet contamination and finally sedimented at $400 \times g$. The resulting pellet was washed with complete EGM-2 then sedimented at $200 \times g$. R\&D UCB MNC were cryopreserved in Hyclone FCS containing $10 \%$ (v/v) DMSO in Nunc cryovials using a Nalgene Mr. Frosty cryopreservation chamber at $-80^{\circ} \mathrm{C}$. After at least $4 \mathrm{~h}$, cryovials were transferred to the gas-phase of liquid nitrogen storage.

UCB destined for banking for unrelated transplants is stored by the NHSBT Cord Blood Bank (termed 'CBB UCB'). The UCB for this purpose was collected in 2004-2005 by methods similar to above and described in detail elsewhere [4]. UCB processing was carried out within $24 \mathrm{~h}$ of UCB collection and differs from the R\&D procedure above in that whole UCB was volume reduced to $21 \mathrm{ml}$ and depleted of plasma and erythrocytes using the Biosafe Sepax system $[3,18]$. The UCB buffy coat was then cryopreserved using DMSO and stored in a BioArchive. $\mathrm{CD} 34^{+}$cells were enumerated by flow cytometry using the ISHAGE protocol. The starting volumes and TNC of CBB UCB units were similar to R\&D UCB units (Table 2). Of the UCB units used here, the majority were made available for research because consent was specifically given to donate to research but not banking (63\%). Other reasons were low TNC (5\%) and failure to complete 12 week follow up interviews necessary for banking (32\%).

\section{Recovery of MNC from cryopreservation}

Cryopreservation vials were removed from gas-phase liquid nitrogen, briefly stored in dry ice and then thawed rapidly at $37^{\circ} \mathrm{C}$ in a waterbath. Without delay, the volume of thawed MNC volume was doubled over 1 min by gradual drop-wise addition of warm $\left(37^{\circ} \mathrm{C}\right)$ thaw buffer with gentle mixing. Five further volumes of warm thaw buffer were then added, mixed gently and incubated at $37^{\circ} \mathrm{C}$ for 30 min with DNase 1 in a sealed tube. Cells were pelleted by centrifugation at $22^{\circ} \mathrm{C}$ for $10 \mathrm{~min}$ at $200 \times g$. Supernatant was aspirated $(90 \%$ of total volume) and cells resuspended by gentle pipetting.

Endothelial cell culture and enumeration of primary endothelial colony forming cells

UCB MNCs were plated at $2 \times 10^{7} \mathrm{MNCs} /$ well in $4 \mathrm{ml}$ complete EGM-2 into rat collagen 1-coated 6-well plates and grown for $24 \mathrm{~h}$ at which time cultures were then washed gently three times with wash buffer B to remove unattached cells and debris, before feeding with complete EGM-2. Growth medium was changed daily for six further
Table 2 Characteristics of UCB

${ }^{a}$ CBB UCB units are volume reduced to $21 \mathrm{ml}$ for processing whereas R\&D UCB units are not. Error is SEM

\begin{tabular}{lll}
\hline Characteristic & CBB UCB & R\&D UCB \\
\hline Volume $(\mathrm{ml})$ & $67 \pm 5$ & $89 \pm 4$ \\
$\mathrm{TNC} \times 10^{7}(/ \mathrm{UCB}$ unit $)$ & $82 \pm 12$ & $77 \pm 1$ \\
$\mathrm{MNC} \times 10^{6}(/ \mathrm{ml})$ & $39.4 \pm 6.0^{\mathrm{a}}$ & $8.1 \pm 0.7$ \\
$\mathrm{CD} 34^{+} \times 10^{6}(/ \mathrm{UCB}$ unit $)$ & $2.7 \pm 0.8$ & $\mathrm{n} / \mathrm{a}$ \\
$\mathrm{n}$ & 19 & 30 \\
\hline
\end{tabular}


days, then every second day from $\mathrm{d} 8$ to $\mathrm{d} 14$. ECFC-derived colonies based on those with endothelial morphology were counted on d14 using light microscopy (Nikon Eclipse TS100, Nikon Ltd., London, England) and contained $>50$ cells/colony. ECFC frequency was enumerated in three ways (A) per UCB unit, (B) per $\mathrm{ml}$ of UCB and (C) normalized per $10^{6} \mathrm{MNC}$ seeded. At this time, these primary colonies were defined as passage $0(\mathrm{p} 0)$, and a passage number incremented with each subsequent trypsination and subculture.

At d14, cultures were gently washed three times with wash buffer B to remove all media and debris and individual colonies isolated by incubation with trypsin-EDTA for 10 min or until completely detached. Where necessary to avoid non-endothelial morphology cells (e.g. single attached cells, striated cells), cloning rings were used to isolate endothelial colonies. ECFC-derived colonies (on average 10 per culture) were pooled and seeded into a T75 flask $\left(75 \mathrm{~cm}^{2}\right.$ surface area) in $30 \mathrm{ml}$ of complete EGM-2. When cells reached approximately $90 \%$ confluency, they were subcultured again as follows. T75 flasks were washed three times with $30 \mathrm{ml}$ of Dulbecco's phosphate wash buffered saline (DPBS) (Lonza Biologics) to remove all residual complete media and serum. Cells were incubated with $3 \mathrm{ml}$ trypsin-EDTA for $10 \mathrm{~min}$ or until they were completely detached from the flask. Cells were split at a dilution rate to give $90 \%$ confluency $4-5$ days later. The endothelial nature of the ECFC-derived cells was confirmed by morphology and phenotypic analysis as described [19].

\section{Proliferative potential of endothelial colony forming} cells

Proliferative potential of ECFC was measured in the clonogenic endothelial colony-formation assay as described previously [19]. Briefly, pooled p1 endothelial colonies (d14) from each UCB harvest were pooled, diluted to give 0.5 cells/well by limiting dilution and manual pipetting in collagen-coated 96-well plates in $100 \mu \mathrm{l}$ complete EGM-2 and grown for 14 days with complete media replacement every 3-5 days. On d14 colonies were examined microscopically and by Calcein AM staining and scored. Primary colonies were scored as previously described as high proliferative potential (HPP) $(>2,000$ cells/colony), low proliferative-potential (LPP) $\quad(50-2,000$ cells/colony or disregarded as endothelial clusters ( $<50$ cells/colony) [19].

\section{Statistical analyses}

Statistical analysis of the effect of obstetric factors consisted of transforming the data to give a normal distribution. In the generalized linear model, framework discriminant analysis was carried out by fitting a binary regression analysis using $\mathrm{R}$ statistical package. Other tests of statistical significance were performed with the software program, Prism (GraphPad, Version 5, San Diego, USA).

\section{Results}

Obstetric and demographic factors analyzed

Obstetric factors have proven useful as selection criteria for UCB units for HSC transplantation. A similar approach was taken here for assessing ECFC content in UCB units in this study. Many factors are known about the mother and baby before the delivery (prenatal), at the time of the delivery (perinatally) and shortly after the delivery at the time of UCB collection, selection and processing (postnatally). Prenatal factors examined were maternal parity, gravidity, age, ethnic group, sex of the baby and gestation period. Perinatal factors examined were the duration of labor and the stages of labor, delivery method, route of delivery, weight of placenta, birth weight, ethnic group of baby and Apgar scores at 1 , 5 and $10 \mathrm{~min}$. Post natal features of UCB were volume of $\mathrm{UCB}, \mathrm{MNC}$ concentration, TNC and nucleated red blood cell concentration.

To test whether obstetric factors had an effect on ECFC yield from UCB, ECFC were enumerated in 52 fresh R\&D UCB units collected at random. Demographic information about the UCB donors is presented in Tables 3 and 4. Of note, those collected for research purposes were dissimilar to national averages for cord blood banking regarding route of delivery, parity, gravidity, placental weight and ethnicity. Of the deliveries, $27 \%$ were vaginal, $71 \%$ were cesarean and $2 \%$ were a cesarean after first stage labor. This statistic reflects the nature of UCB collection of UCB units for research at the John Radcliffe Hospital, where cesarean sections present the best prospect for collecting UCB due to the increased opportunity for pre delivery written informed consent, the relatively predictable timeframe for delivery and availability of the placenta, and lower frequency of damage to afterbirth tissues. Parity and gravidity were higher than the national averages. Mean placental weight exceeded the UK average for babies born at term which is $\sim 545 \mathrm{~g}$, and reflected the fact that the majority of births were by cesarean section which tend to result in higher placental weights [20]. Sixty-one percent of babies were male, and $39 \%$ were female. Ninety percent of births were from white Caucasoid mothers. Many other demographic features were similar to the UK national averages. Maternal age was similar to the UK national average of 29.3 years [20] and neonatal weight was similar to the average UK birth weight of approximately $3,400 \mathrm{~g}$ [20]. As dictated by the scope of this study, all babies in 
Table 3 Demographics of prenatal and perinatal maternal, obstetric and neonatal observations of UCB donors

\begin{tabular}{|c|c|c|c|}
\hline Characteristics (n) & Range & Number & Percent $(\%)$ \\
\hline \multirow[t]{3}{*}{ Maternal age (years) (52) } & $18-25$ & 8 & 15.4 \\
\hline & $26-35$ & 30 & 57.7 \\
\hline & 35 & 14 & 26.9 \\
\hline \multirow[t]{4}{*}{ Parity (49) } & 0 & 13 & 26.5 \\
\hline & 1 & 18 & 36.7 \\
\hline & 2 & 15 & 30.6 \\
\hline & $\geq 3$ & 3 & 6.1 \\
\hline \multirow[t]{4}{*}{ Gravidity (49) } & 0 & 30 & 61.2 \\
\hline & 1 & 11 & 22.4 \\
\hline & 2 & 6 & 12.2 \\
\hline & $\geq 3$ & 2 & 4.1 \\
\hline \multirow[t]{2}{*}{ Mode of delivery (48) } & Vaginal & 13 & 27.1 \\
\hline & Caesarean $^{\mathrm{a}}$ & 35 & 72.9 \\
\hline \multirow[t]{2}{*}{ Neonatal sex (49) } & Male & 30 & 61.2 \\
\hline & Female & 19 & 38.8 \\
\hline \multirow[t]{5}{*}{ Apgar 1 min (48) } & $<7$ & 1 & 2.0 \\
\hline & 7 & 0 & 0.0 \\
\hline & 8 & 5 & 10.4 \\
\hline & 9 & 14 & 29.2 \\
\hline & 10 & 28 & 58.3 \\
\hline \multirow[t]{5}{*}{ Apgar 5 min (48) } & $<7$ & 1 & 2 \\
\hline & 7 & 0 & 0 \\
\hline & 8 & 0 & 0 \\
\hline & 9 & 0 & 0 \\
\hline & 10 & 47 & 97.9 \\
\hline \multirow[t]{5}{*}{ Apgar $10 \min (48)$} & $<7$ & 0 & 0 \\
\hline & 7 & 0 & 0 \\
\hline & 8 & 0 & 0 \\
\hline & 9 & 0 & 0 \\
\hline & 10 & 48 & 100 \\
\hline \multirow[t]{2}{*}{ Ethnic group (49) } & White & 44 & 89.8 \\
\hline & Other & 5 & 8.2 \\
\hline
\end{tabular}

a 34 caesarean without labor, 1 caesarean after 1st stage labor

this study were born at term with gestational age ranging from 37 to 41 weeks. Only $3.3 \%$ of babies had an Apgar $_{1 \min }$ score $<7$, indicating the majority of neonates in this study were in overall good health.
Table 5 Endothelial colony forming cell (ECFC) content of umbilical cord blood units

\begin{tabular}{lcl}
\hline Characteristics & Mean \pm SEM & Range \\
\hline ECFC/UCB unit & $44.6 \pm 9.8$ & $0-375$ \\
ECFC/ml & $0.5 \pm 0.1$ & $0-4.1$ \\
ECFC/MNC $\left(\times 10^{8}\right)$ & $8.2 \pm 1.8$ & $0-57.1$ \\
\hline
\end{tabular}

$\mathrm{n}=52 \mathrm{UCB}$ units

Placental weight predicts ECFC content of UCB units

Table 5 summarizes the mean numbers and the range of ECFC found in the $52 \mathrm{UCB}$ units tested. ECFC-derived cells were confirmed by morphology and phenotype as endothelial in nature (Fig. 1). The range of ECFC frequencies was from 0 to $375 \mathrm{ECFC/unit}$ with $8 \mathrm{UCB}$ units not forming ECFC and with the mean ECFC numbers being $44.6 \pm 9.8 \mathrm{ECFC} / \mathrm{UCB}$ unit or $8.2 \pm 1.8 \mathrm{ECFC} / 10^{8} \mathrm{MNC}$.

In testing the effect of obstetric factors, skewed data were transformed by taking the Box-Cox transformation to achieve an approximate normal distribution of the data and to stabilize the variance, thereby satisfying the conditions for a valid linear model. Some of the explanatory variables were also transformed to remove excessive skewness. Fitting of a linear model regression was then attempted for the full data set $(n=52)$ initially using ECFC/UCB as a unit of measurement of ECFC frequency. This was because, of the three experimental outcomes examined for ECFC frequency (per unit, per ml UCB, per $10^{8} \mathrm{MNC}$ ), ECFC/UCB unit responded best to transformation i.e. achieved the best approximation of a normal distribution of the data. The effect of multiple obstetric factors was examined starting with all obstetric variables and the least significant variables eliminated from the modeling in a stepwise fashion. Neither combination of the obstetric factors nor any single factor exhibited a statistically significant causal relationship with ECFC frequency/UCB unit. Similar outcomes were obtained from the same analyses with $\mathrm{ECFC} / \mathrm{ml} \mathrm{UCB}$ and $\mathrm{ECFC} / 10^{8} \mathrm{MNC}$.

An alternative modeling approach was taken by excluding all UCB where EFCF/UCB unit $=0$ and analyzing the remaining data using the approach above. With the zeroes omitted, it was possible to find the optimal BoxCox transformation of the response variable, which had the
Table 4 Demographics of perinatal maternal, obstetric and neonatal variables

\begin{tabular}{lcc}
\hline Characteristics (n) & Mean \pm SD & Range \\
\hline Gestational age (weeks) (49) & $39 \pm 1$ & $37-41$ \\
Neonatal weight (g) (49) & $3427 \pm 472$ & $2,620-4,442$ \\
Placental weight (g) (46) & $704 \pm 144$ & $450-1,100$ \\
Time between onset of stage 1 labor and onset of stage 2 labor (h) (13) & $4.4 \pm 0.7$ & $1.3-9.2$ \\
Time between onset of stage 2 labor and delivery of the baby (h) (13) & $0.8 \pm 0.3$ & $0.1-3.1$ \\
Time between delivery of the baby and end of stage 3 labor (h) (13) & $0.2 \pm 0.1$ & $0.0-1.0$ \\
\hline
\end{tabular}



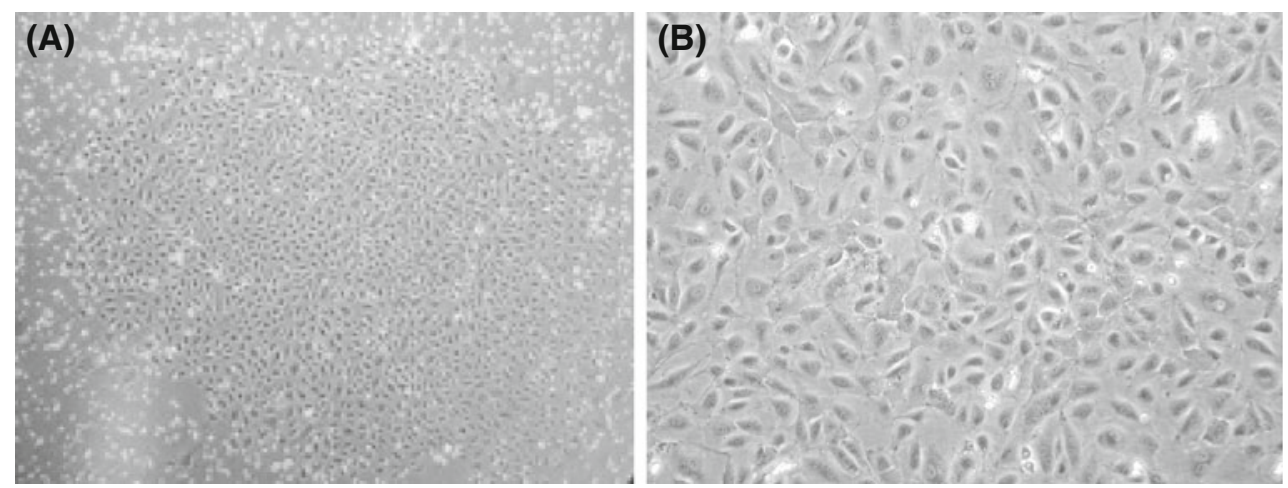

(C)

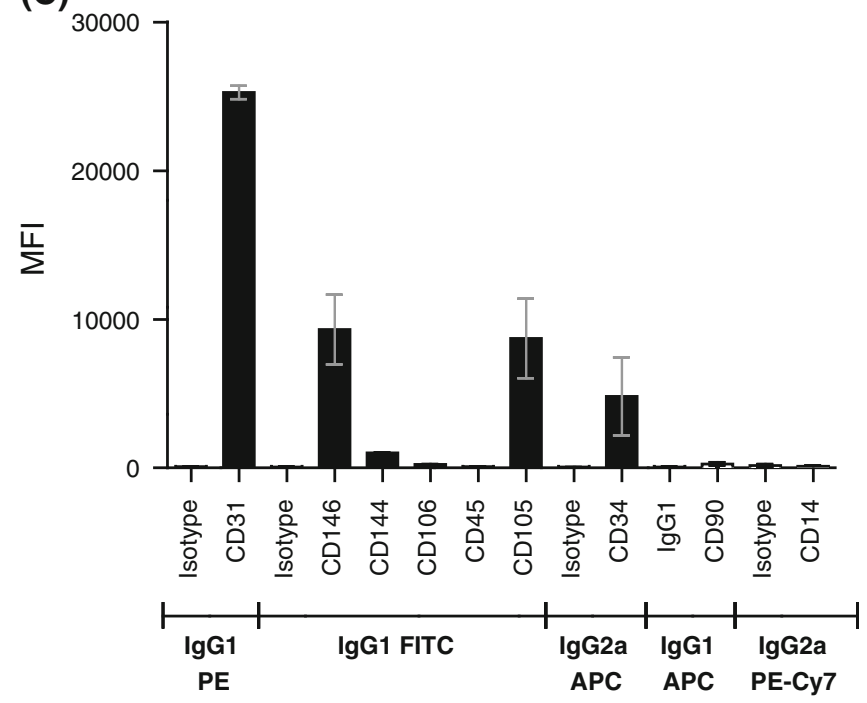

Fig. 1 ECFC-derived cell characteristics. Representative photomicrographs of ECFC-derived colonies cultured from UCB MNCs on a d9 of culture ( $\times 4$ magnification) and $\mathbf{b} \mathrm{d} 14(\times 10$ magnification $)$ of culture. Colonies with similar cobblestone morphology were identified in other cultures of UCB. c Flow cytometric profile of ECFC-

form $\left(\mathrm{Y}^{\wedge}\right.$ lambda -1$) /$ lambda, with lambda $=0.1$. Placental weight (range $450-110 \mathrm{~g}$ ) was the only statistically significant predictor of ECFC frequency $(P$-value $=$ 0.046). No other obstetric factor gave an indication of having a significant effect (Fig. 2). The following formula if $\mathrm{E}_{\text {pred }}$ is predicted ECFC/cord blood unit and $\mathrm{P}$ is placental weight $(\mathrm{g})$ was devised to predict the ECFC frequency from placental weight.

$\mathrm{E}_{\text {pred }}=\left(1.2421+\frac{3.226 \times 10^{3}}{\mathrm{P}^{2 / 3}}\right)^{10}$

The function is plotted in Fig. 2 along with 95\% prediction interval bands and the measured data points with placental weights lower than 2,000 $\mathrm{g}$ have also been added. The range of placental weights was restricted to being less than $2,000 \mathrm{~g}$ in order to avoid undue compression of the scale. It is worth noting that the heavier placental weights in fact turn out to lie almost exactly on the prediction curve. derived cells. Representative graph of median fluorescence intensity $(\mathrm{MFI})+$ SEM $(\mathrm{n}=3)$ using cells from different donors. Antibody isotypes and fluorophores are grouped to compare test antibody staining with isotype control staining

Further, a discriminant analysis approach was taken by awarding a binary variable to ECFC/UCB unit and a fit of a generalized linear model attempted, i.e. UCB units which gave rise to ECFC were awarded a value of one, and those that did not were awarded a value of zero. As above, the effect of multiple obstetric factors was examined starting with all obstetric variables and the least significant variables eliminated from the modeling in a stepwise fashion. By this method, neither combination of the obstetric factors nor any single factor exhibited a statistically significant causal relationship with ECFC frequency/UCB unit.

\section{UCB storage temperatures affect ECFC yield}

In addition to examining obstetric factors, we also assessed if UCB storage temperature affected ECFC yield. Since, in accordance with FACT-Netcord standards, we store fresh CBB UCB units for transplantation from the NHS Cord 


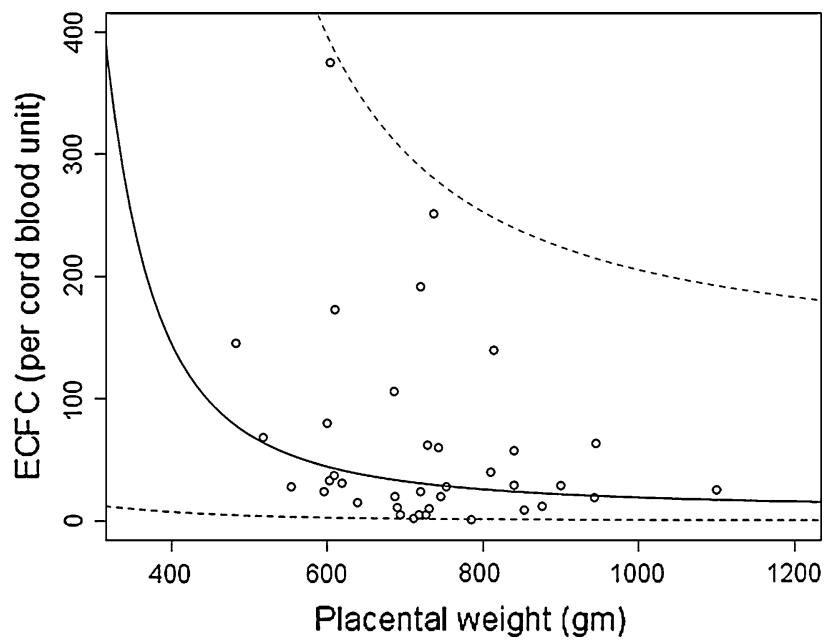

Fig. 2 Correlation between placental weight and ECFC. Model fit (solid line) of the correlation between placental weight and ECFC/ UCB unit $(P<0.05), 95 \%$ confidence intervals are indicated by dashed lines

Blood Bank at $22 \pm 2{ }^{\circ} \mathrm{C}$ after collection and do so for up to $24 \mathrm{~h}$ before processing by volume reduction [18, 21], we examined the effects of transient storage of UCB on ECFC yield. R\&D UCB units were collected from 25 subjects at random and stored at either $4 \pm 2^{\circ} \mathrm{C}$ or $22 \pm 2^{\circ} \mathrm{C}$ in temperature mapped fridges/incubators for $24 \mathrm{~h}$, then the 'Enumeration of ECFC' procedure was followed as detailed in the "Materials and methods".

The data were clearly non-normal and therefore a nonparametric Wilcoxon rank sum test for location difference was carried out with allowance for one pair of tied values in the $4^{\circ} \mathrm{C}$ group. The $P$-value was 0.0013 and it was therefore concluded that there was a highly significant difference between the two groups. Storage for $24 \mathrm{~h}$ at $22^{\circ} \mathrm{C}$ yielded 4 times more ECFC/CBU than storage for $24 \mathrm{~h}$ at $4^{\circ} \mathrm{C}$ (the ratio of medians was 4.07 , the ratio of means was 4.06) (Fig. 3).

The effect of cryopreservation on ECFC was also examined by comparing ECFC yield from fresh R\&D UCB units with their recovery after cryopreservation. To test recovery of ECFC from cryopreservation, $\mathrm{MNC}$ were isolated from $20 \mathrm{R} \& \mathrm{D}$ UCB units as per the "ECFC enumeration' procedure in the "Materials and methods". At the cell pellet stage, before seeding into a 6-well plate, the MNC were split into two samples, one was cryopreserved and the other tested for ECFC as per the 'Enumeration of primary Endothelial Colony Forming Cells' procedure. The cryopreserved sample was stored at $-195^{\circ} \mathrm{C}$ for $2-14$ days, and then thawed in the presence of DNase 1 which was used to digest extracellular DNA from lysed neutrophils, and viable MNC numbers determined. MNC were typically $>95 \%$ viable. Viable MNC were seeded as per the 'Enumeration of Endothelial Colony Forming Cells' procedure
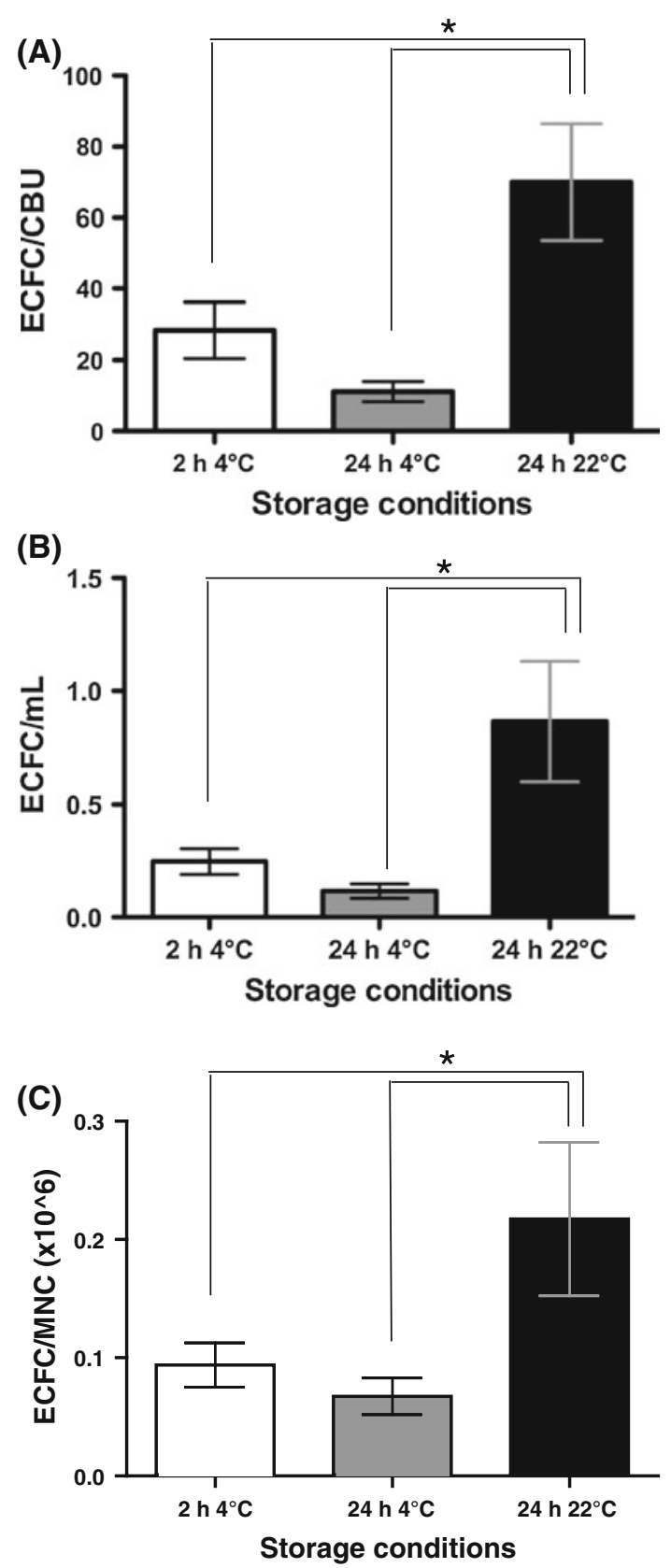

Fig. 3 Effects of short-term storage conditions on yield of ECFC. $\mathrm{UCB}$ were stored for either $2 \mathrm{~h}$ at $4^{\circ} \mathrm{C}(\mathrm{n}=6), 24 \mathrm{~h}$ at $4^{\circ} \mathrm{C}(\mathrm{n}=9)$ or $24 \mathrm{~h}$ at $22^{\circ} \mathrm{C}(\mathrm{n}=10)$. Error bars are SEM. ECFC yields were assessed per a UCB unit, b $\mathrm{ml}$ UCB and c MNC $\left(\times 10^{6}\right)$ seeded. Wilcoxon statistical analysis showed that storage at $4^{\circ} \mathrm{C}$ had a significant effect on ECFC yield both for 2 and $24 \mathrm{~h}(* P=0.0013)$

and the resulting ECFC content of the cryopreserved UCB compared with ECFC yield from fresh UCB. Ninety percent of fresh R\&D UCB samples generated ECFC compared to $33 \%$ of the cryopreserved UCB samples. When the latter $33 \%$ of UCB units that formed ECFC in both fresh and frozen UCB samples were compared, the ECFC recovery from cryopreservation was $\sim 50 \%$ lower than that found in the original fresh UCB sample (Fig. 4b). The 

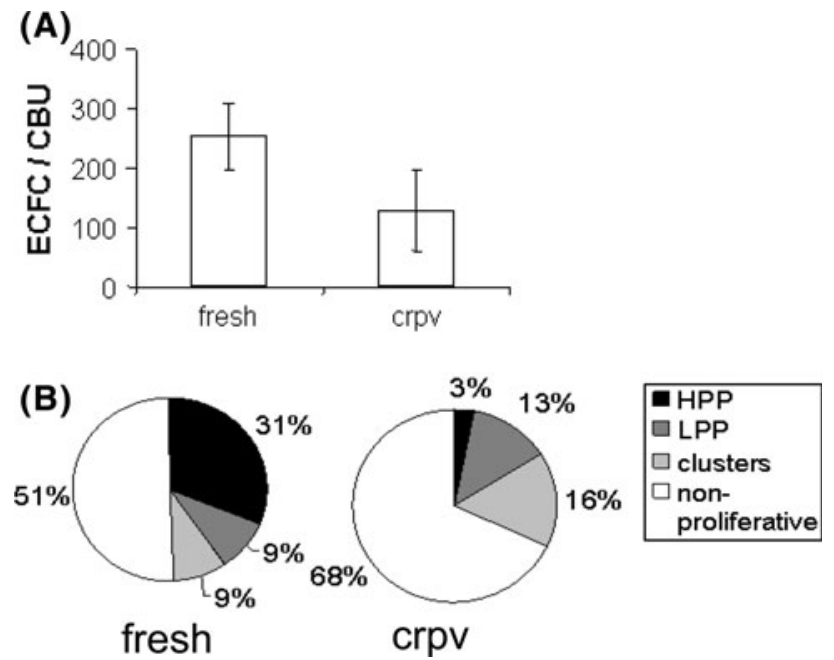

Fig. 4 UCB ECFC recovery from cryopreservation. a Recovery of ECFC from cryopreservation compared with yield from fresh UCB (fresh $\mathrm{n}=23$, crpv $\mathrm{n}=15$, error bars are SEM). b Proliferative potential of recovered ECFC from cryopreservation compared with ECFC proliferative potential from fresh UCB (fresh $\mathrm{n}=8$, crpv $\mathrm{n}=3$ ); crpv cryopreserved; LPP, low proliferative potential cells [19]; HPP, high proliferative potential cells [19]

quality of ECFC was also investigated by examining the proliferative potential of the ECFC-derived cells in a clonal proliferation assay. Endothelial colonies derived from each UCB MNC preparation before and after cryopreservation from the previous experiment were pooled and cultured (p2) and their content of ECFCs which formed colonies of different sizes assessed in the clonogenic assay described in 'Proliferative potential' in the Materials and Methods.

ECFC yield from $\mathrm{CBB}$ procedures were examined by enumerating ECFC from cryopreserved CBB UCB units and the results presented in Fig. 5. ECFC yield/ml UCB was sixfold lower in CBB than cryopreserved R\&D UCB units indicating ECFC losses during CBB UCB processing procedures (Fig. 5a). Interestingly, of those ECFC a threefold higher proportion of these were high-proliferative and a 1.4-fold higher proportion were low-proliferative. The remainder (clusters and non-proliferative) was 1.2-fold higher in R\&D than CBB UCB (Fig. 5b). However these findings conflict with the absolute levels of HPP and LPP per volume of UCB (considering CBB UCB exhibited sixfold lower $\mathrm{ECFC} / \mathrm{ml}$ ). Collectively these findings suggest that, qualitatively and quantitatively, $C B B$ and $R \& D$ UCB units are comparable in terms of highly proliferative cells. However, there may be the possibility of increasing ECFC yields, and hence highly proliferative ECFC yields, from CBB UCB units as indicated by ECFC/ml UCB.

In $\mathrm{CBB}$ UCB units, the relationship between $\mathrm{CD} 34^{+}$cell numbers and ECFC was examined by testing for a linear correlation between $\mathrm{CB} 34^{+}$cells per UCB unit and ECFC/
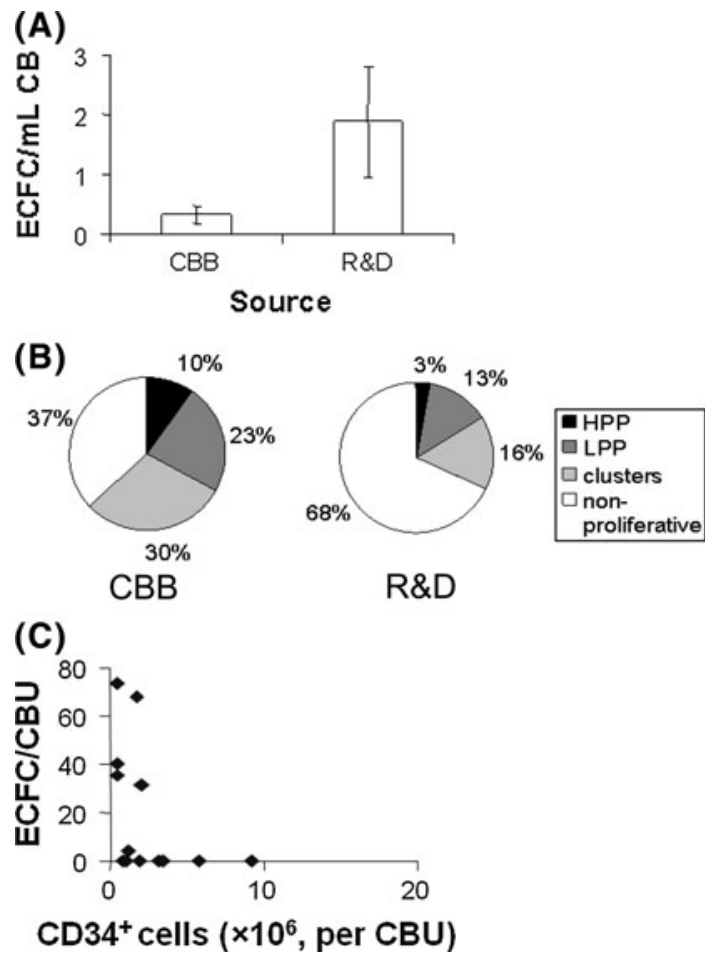

Fig. 5 UCB ECFC recovery from cryopreserved R\&D and CBB sources. a ECFC frequency in UCB $(\mathrm{CBB} n=14$, R\&D $n=15$, error bars are SEM). b Proliferative potential of ECFC by source (CBB $\mathrm{n}=3, \mathrm{R} \& \mathrm{D} \mathrm{n}=3$ ). $\mathbf{c} \mathrm{ECFC}$ frequency/cord blood unit versus CD $34^{+}$ cells/UCB unit. $C B B$ cord blood bank-sourced UCB units; $R \& D$ research resource-sourced UCB units; $C B U$ cord blood unit; LPP, low proliferative potential cells; HPP, high proliferative potential cells

UCB unit (Fig. 5c). No correlation was observed $\left(\mathrm{r}^{2}=\right.$ 0.1490 ) suggesting that $\mathrm{CD} 34^{+}$cell content in UCB units would not be good predictor of ECFC content.

\section{Discussion}

The therapeutic potential for ECFC for supporting blood cell reconstitution, vascular engineering, neovasuclarisation and cardiovascular repair has been widely discussed [8]. In order to fully realize the clinical utility of ECFCs that are known to be present in UCB, we must first understand factors affecting the yield of these cells from UCB. The study presented here focused on whether obstetric factors, transient pre-processing storage and longterm cryopreserved storage could influence the ECFC content of UCB at term, areas which have not previously been fully investigated. Our results demonstrate a positive correlation between placental weight and ECFC content of UCB in the donor population studied. Previous studies have shown that placental weight and weight of the infant at birth positively correlate with TNC count, UCB volume, $\mathrm{CD} 4^{+}$cell content and total hematopoietic CFU in UCB (Table 1). Indeed, Ballen et al. [22] have suggested that 
each $500 \mathrm{~g}$ increase in birth weight contributes to a $28 \%$ increase in $\mathrm{CD}_{3} 4^{+}$cell counts.

However, CD34 has suggested to be both a hematopoietic and endothelial progenitor and mature endothelial marker [8-10], hence, it has been difficult to correlate $\mathrm{CD} 34^{+}$cell numbers in peripheral blood or UCB with ECFC content in normal individuals. ECFCs are thought to represent a minor proportion of the $\mathrm{CD} 34^{+}$cells which do not express CD45 [8, 15, 23, 24]. For the CBB UCB units used here, the ISHAGE protocol for $\mathrm{CD} 34^{+}$enumeration reported total $\mathrm{CD} 34^{+} \mathrm{CD} 45^{\mathrm{dim}}$ which probably do not best represent ECFC. Indeed, in the present study, we observed no correlation between $\mathrm{CD} 34^{+}$expressing cells and ECFC frequency. Currently, UCB banks select UCB units for HSC transplant based on $\mathrm{CD} 34^{+}$cell content thresholds and the requirement for maximal ethnic diversity. However, in light of the current findings, UCB units reported as having low $\mathrm{CD} 34^{+}$content according to the ISHAGE protocol may still be a useful source of ECFC and so should not be discarded.

We saw no correlations with many of the obstetric factors examined including parity, mode of delivery or infant gender. As the donor population was skewed towards uncomplicated pregnancies and deliveries at term from Caucasoid mothers, an analysis of a wider range of Apgar scores, gestational ages and ethnicity on ECFC content of UCB was not possible in our study. It was not possible to analyze the effect of ethnic origin on ECFC content of UCB in this study, yet this is a factor of particular relevance to UCB banking especially in the UK where adult bone marrow registries lack ethnic diversity of donors. Ethnicity has been shown to have no significant effect on TNC in relation to HSC/HPC UCB banking and transplantation [25] while Caucasoid births produce higher $\mathrm{UCB} \mathrm{CD} 34^{+}$yields [26]. In those ethnic groups where placental weight is lower than in white Caucasoid donors [4], the ECFC numbers may well be lower than in term UCB units from larger white Caucasoid donors.

Interestingly, our studies have allowed us to develop a formula to predict the ECFC frequency from placental weight with a $95 \%$ confidence interval for the predicted value and this formula could be used in the future with defined ethnic groups to confirm its robustness. Baker et al. [15] have suggested that ECFC content and proliferative ability in UCB may contribute to organogenesis in for example the renal glomerulus and retina and the need to significantly expand the pulmonary microvasculature during late gestation and early post natal life. It would thus seem reasonable to assume that the rate of development may also correlate with the UCB content of ECFC and proangiogenic hematopoietic cells, both of which have key roles in neovasculogenesis [8, 24].

In addition to assessing obstetric factors, we also analyzed the effects of transient storage temperatures on ECFC yield from UCB. Current FACT-Netcord standards support transient storage and transport of UCB units for subsequent HSC transplantation prior to processing at $22 \pm 2^{\circ} \mathrm{C}[1,2$, 4]. Our own practice at the NHS Cord Blood Bank is to maintain UCB units at these temperatures for up to $24 \mathrm{~h}$ before processing is initiated [27]. This is because initial studies involving human HPCs demonstrated that UCB storage at both $4^{\circ} \mathrm{C}$ and room temperature for up to $24 \mathrm{~h}$ led to little or no cell loss [29-31], with slightly higher counts for UCB stored at room temperature [29] and so, consensus is that there is no significant effect on TNC and $\mathrm{CD} 34^{+}$cell viability and hematopoietic CFU when UCB is stored at room temperature for $24 \mathrm{~h}$. However, storage conditions for longer times are conflicting. Recent studies [32] suggest that, with the exception of viable $\mathrm{CD} 34^{+}$cells, there is a significant decrease in TNC, MNC and CD45 ${ }^{+}$ cell viability between 72 and $96 \mathrm{~h}$ and, with the exception of viable $\mathrm{CD}_{3} 4^{+}$cells and CFU-GM, the reductions are significantly higher with room temperature as opposed to $4^{\circ} \mathrm{C}$ storage pre-processing. Our studies revealed that we could recover higher numbers of ECFC if UCB was stored at $22 \pm 2^{\circ} \mathrm{C}$ as opposed to $4^{\circ} \mathrm{C}$ storage for $24 \mathrm{~h}$. Our findings may be explained by the fact that storage at room temperature instead of refrigeration avoids rapid cooling and subsequent warming of the blood which leads to temperature shock and can trigger cellular apoptosis [14]. To our knowledge, similar studies concerning ECFC recovery have not been done.

In agreement with Vanneaux et al. [17], our studies indicate that cryopreservation of UCB MNCs, even after room temperature storage for up to $24 \mathrm{~h}$, significantly affected ECFC recovery. However, the quantity of HPP ECFCs was less affected by cryopreservation than the overall ECFC yield from UCB. If proliferative potential of ECFC is of clinical importance in future therapeutic applications, then indeed HPP ECFC yield is the more important criteria.

As well as the limitations described above, this and similar studies are also limited by the lack a specific biomarker for enumerating ECFC in UCB, particularly in light of the lack of a correlation observed with CD34. An important question from the clinical perspective is: 'how many ECFCs are required for a useful therapeutic dose?' which raises the possibility of expanding and banking ECFC from UCB as a further transplant option. We are still some way from using banked UCB units as a source of ECFCs in the clinic where they may promote hematopoietic engraftment and tissue repair. However, UCB transplantation and UCB banking are both rapidly evolving fields and in future, any readily available information regarding the efficacy of stem/progenitor cells in such units may impact significantly on the quality of units selected for transplantation and research. With this in mind, further 
studies on more diverse populations of donors are warranted to fully determine which obstetric factors affect ECFC numbers in UCB.

Acknowledgments This work presents independent research commissioned by the National Institute for Health Research (NIHR) under its Program Grants scheme. The views expressed in this publication are those of the author (s) and not necessarily those of the NHS, the NIHR or the Department of Health. This work was supported by research funding from NHS Blood and Transplant (GT, JS, and SMW), National Institutes of Health Research (SMW, GT, and CPK), Restore Burn and Wound Research (JK, SMW), the Cord Blood Charity (KEC, SMW, JS), the University of Melbourne (SJL) and the EU Framework VII Cascade grant (SMW). JK is a recipient of a Duke of Kent Research Fellowship. The sponsors of this study are public, non profit organizations that support science in general. They had no role in gathering, analyzing or interpreting the data. The authors wish to acknowledge Dr D Lunn of the Oxford University Statistical Consultancy Service, who carried out the statistical analyses, Mrs. Sandra Britt and Ms Janice Gotobed, who collected the R\&D UCB units and the staff of the NHS Cord Blood Bank for providing CBB UCB units.

Open Access This article is distributed under the terms of the Creative Commons Attribution Noncommercial License which permits any noncommercial use, distribution, and reproduction in any medium, provided the original author(s) and source are credited.

\section{References}

1. Watt SM (2010) Umbilical cord blood stem cell banking. In: Mood-Young M (ed) Comprehensive biotechnology. Elsevier B.V, Kidlington

2. Watt SM (2010) Business models of cord blood. In: Prescott C, Pollack J (eds) Delivery of regenerative medicines and their impact on healthcare. Taylor and Francis Group, London

3. Smythe J et al (2007) Directed sibling cord blood banking for transplantation: the 10-year experience in the national blood service in England. Stem Cells 25(8):2087-2093

4. Watt S, Coldwell K, Smythe J (2010) Comparisons between related and unrelated cord blood collection and/or banking for transplantation or research: the UK NHS blood and transplant experience. In: Bhattacharya $\mathrm{N}$ (ed) Clinical use of placenta, amniotic fluid, umbilical cord and its contents: current medical perspectives. Springer, London

5. NHS Blood and Transplant UK Stem Cell Strategic Forum, The future of unrelated donor stem cell transplantation in the UK: Part 1 Findings and Recommendations, 2010

6. Salter AB et al (2009) Endothelial progenitor cell infusion induces hematopoietic stem cell reconstitution in vivo. Blood 113(9):2104-2107

7. English K, French A, Wood KJ (2010) Mesenchymal stromal cells: facilitators of successful transplantation? Cell Stem Cell 7(4):431-442

8. Watt $\mathrm{S}$ et al (2010) Human endothelial stem-progenitor cells, angiogenic factors and vascular repair. J R Soc Interface 7:S731

9. Au P et al (2008) Differential in vivo potential of endothelial progenitor cells from human umbilical cord blood and adult peripheral blood to form functional long-lasting vessels. Blood 111(3):1302-1305

10. Ingram DA, Caplice NM, Yoder MC (2005) Unresolved questions, changing definitions, and novel paradigms for defining endothelial progenitor cells. Blood 106(5):1525-1531
11. Wagner JE, Gluckman E (2010) Umbilical cord blood transplantation: the first 20 years. Semin Hematol 47(1):3-12

12. Barker JN, Byam C, Scaradavou A (2010) How we search: a guide to the selection and acquisition of unrelated cord blood grafts. Blood 117(8):2332-2339

13. Avery $S$ et al (2010) Influence of infused cell dose and HLAmatch on engraftment after double-unit cord blood allografts. Blood 117(12):3277-3285

14. Querol S et al (2010) Quality rather than quantity: the cord blood bank dilemma. Bone Marrow Transp 45(6):970-978

15. Baker CD et al (2009) Endothelial colony-forming cells from preterm infants are increased and more susceptible to hyperoxia. Am J Respir Crit Care Med 180(5):454-461

16. Lin Y et al (2000) Origins of circulating endothelial cells and endothelial outgrowth from blood. J Clin Invest 105(1):71-77

17. Vanneaux $\mathrm{V}$ et al (2010) In vitro and in vivo analysis of endothelial progenitor cells from cryopreserved umbilical cord blood: are we ready for clinical application? Cell Transp 19(9):1143-1155

18. Watt SM, Austin E, Armitage S (2007) Cryopreservation of hematopoietic stem/progenitor cells for therapeutic use. Methods Mol Biol 368:237-259

19. Zhang $Y$ et al (2009) The impact of proliferative potential of umbilical cord-derived endothelial progenitor cells and hypoxia on vascular tubule formation in vitro. Stem Cells Dev 18(2): 359-375

20. Burkhardt $\mathrm{T}$ et al (2006) Reference values for the weight of freshly delivered term placentas and for placental weight-birth weight ratios. Eur J Obstet Gynecol Reprod Biol 128(1-2): 248-252

21. Davey S et al (2004) The London cord blood bank: analysis of banking and transplantation outcome. Br J Haematol 125(3): 358-365

22. Ballen KK et al (2001) Bigger is better: maternal and neonatal predictors of hematopoietic potential of umbilical cord blood units. Bone Marrow Transpl 27(1):7-14

23. Acosta JC et al. (2010) Gestational diabetes mellitus alters maternal and neonatal circulating endothelial progenitor cell subsets. Am J Obstet Gynecol 204(3):254e8-254e15

24. Critser PJ, Yoder MC (2010) Endothelial colony-forming cell role in neoangiogenesis and tissue repair. Curr Opin Organ Transpl 15(1):68-72

25. Ballen KK et al (2004) Racial diversity with high nucleated cell counts and CD34 counts achieved in a national network of cord blood banks. Biol Blood Marrow Transpl 10(4):269-275

26. Cairo MS et al (2005) Characterization of banked umbilical cord blood hematopoietic progenitor cells and lymphocyte subsets and correlation with ethnicity, birth weight, sex, and type of delivery: a Cord Blood Transplantation (COBLT) Study report. Transfusion 45(6):856-866

27. Pamphilon DH, Selogie E, Szczepiorkowski ZM (2010) Transportation of cellular therapy products: report of a survey by the cellular therapies team of the biomedical excellence for safer transfusion (BEST) collaborative. Vox Sang 99(2):168-173

28. Juutistenaho $S$ et al (2010) Association of stress-related perinatal factors and cord blood unit hematopoietic progenitors is dependent on delivery mode. Transfusion 50(3):663-671

29. Broxmeyer HE et al (1989) Human umbilical-cord blood as a potential source of transplantable hematopoietic stem progenitor cells. Proc Natl Acad Sci USA 86(10):3828-3832

30. Case J, Rice A, Vowels M (1996) Conditions affecting the isolation of human umbilical cord blood CD34+ cells. J Hematother 5(3):255-260

31. Hubel A et al (2003) Cryopreservation of cord blood after liquid storage. Cytotherapy 5(5):370-376

32. Pamphilon D et al (2010) Storage characteristics of cord blood progenitor cells: report of a multicenter study by the cellular 
therapies team of the Biomedical Excellence for Safer Transfusion (BEST) Collaborative. Transfusion 51(6):1284-1290

33. Askari $S$ et al (2005) Impact of donor- and collection-related variables on product quality in ex utero cord blood banking. Transfusion 45(2):189-194

34. McGuckin CP et al (2007) Cord blood revelations: the importance of being a first born girl, big, on time and to a young mother!. Early Hum Dev 83(12):733-741

35. Aroviita P et al (2005) Cord blood hematopoietic progenitor cell concentration and infant sex. Transfusion 45(4):613-621

36. Mancinelli F et al (2006) Optimizing umbilical cord blood collection: impact of obstetric factors versus quality of cord blood units. Transpl Proc 38(4):1174-1176

37. Nakagawa R et al (2004) Analysis of maternal and neonatal factors that influence the nucleated and CD34+ cell yield for cord blood banking. Transfusion 44(2):262-267

38. Solves $\mathrm{P}$ et al (2004) Optimizing donor selection in a cord blood bank. Eur J Haematol 72(2):107-112

39. Solves $\mathrm{P}$ et al (2005) Maternal, neonatal and collection factors influencing the haematopoietic content of cord blood units. Acta Haematol 113(4):241-246

40. Jones $\mathrm{J}$ et al (2003) Obstetric predictors of placental/umbilical cord blood volume for transplantation. Am J Obstet Gynecol 188(2):503-509

41. Urciuoli P et al (2010) Pre-birth selection of umbilical cord blood donors. Blood Transfus 8(1):36-43
42. Mohyeddin Bonab MA, Alimoghaddam KA, Goliaei ZA, Ghavamzadeh AR (2004) Which factors can affect cord blood variables? Transfusion 44:690-693

43. Aroviita $\mathrm{P}$ et al (2004) Birthweight of full-term infants is associated with cord blood CD34+ cell concentration. Acta Paediatr 93(10):1323-1329

44. Dimitriou $\mathrm{H}$ et al (2006) The impact of mode of delivery and gestational age on cord blood hematopoietic stem/progenitor cells. Ann Hematol 85(6):381-385

45. Lim FT et al (2000) Association of stress during delivery with increased numbers of nucleated cells and hematopoietic progenitor cells in umbilical cord blood. Am J Obstet Gynecol 183(5):1144-1152

46. Sparrow RL et al (2002) Influence of mode of birth and collection on WBC yields of umbilical cord blood units. Transfusion 42(2):210-215

47. Screnci M et al. (2009) Related cord blood banking for haematopoietic stem cell transplantation. Transfus Med 20(3):185-190

48. Lim FT et al (1994) Influence of delivery on numbers of leukocytes, leukocyte subpopulations, and hematopoietic progenitor cells in human umbilical cord blood. Blood Cells 20(2-3): 547-558 (discussion 558-9)

49. Ingram DA et al (2008) In vitro hyperglycemia or a diabetic intrauterine environment reduces neonatal endothelial colonyforming cell numbers and function. Diabetes 57(3):724-731 